\title{
Scanning drift tube measurements of electron transport parameters in different gases: argon, synthetic air, methane and deuterium
}

\author{
I Korolov, M Vass and Z Donkó \\ Institute for Solid State Physics and Optics, Wigner Research Centre for Physics, Hungarian Academy of \\ Sciences, 1121 Budapest, Konkoly Thege Miklós str. 29-33, Hungary \\ E-mail: donko.zoltan@wigner.mta.hu
}

Received 16 May 2016, revised 5 July 2016

Accepted for publication 20 July 2016

Published 13 September 2016

\begin{abstract}
Measurements of transport coefficients of electrons in a scanning drift tube apparatus are reported for different gases: argon, synthetic air, methane and deuterium. The experimental system allows the spatio-temporal development of the electron swarms ('swarm maps') to be recorded and this information, when compared with the profiles predicted by theory, makes it possible to determine the 'time-of-flight' transport coefficients: the bulk drift velocity, the longitudinal diffusion coefficient and the effective ionization coefficient, in a well-defined way. From these data, the effective Townsend ionization coefficient is determined as well. The swarm maps provide, additionally, direct, unambiguous information about the hydrodynamic/ non-hydrodynamic regimes of the swarms, aiding the selection of the proper regions applicable for the determination of the transport coefficients.
\end{abstract}

Keywords: electron swarm, drift tube, transport coefficients

(Some figures may appear in colour only in the online journal)

\section{Introduction}

The transport coefficients of electrons in various gases are fundamentally important quantities and serve as principal input data of widely used fluid models of gas discharges. In addition, this-due to the fact that these coefficients can accurately be calculated from the cross section data-reliable transport coefficient data provide a possibility for checking/ adjusting the electron-neutral collision cross section sets [1].

Throughout the past decades most of the transport coefficient data have been measured using drift tube apparatuses, in which low-density particle 'clouds' or 'swarms' are created and move under the effect of a homogeneous electric field. These systems have been based on different operating principles (photoelectric pulsed drift tubes, double-shutter drift tubes, systems based on photoionization of the gas, etc), see, e.g. [2-6]. As to the detection of the particles, in part of the systems the displacement current generated in the electrode gap by the moving charges [7-9] is sensed, while in other systems the counting of particles arriving at a detector has been employed [3, 10, 11].

Notwithstanding the extensive work carried out in the past, interest in further drift tube measurements still persists. Experiments have been carried out recently for gases like $\mathrm{CF}_{4}$ [12], $\mathrm{CF}_{3} \mathrm{I}$ [13], and tetraethoxysilane [14], which have become important in processing applications. Other gases, like $\mathrm{SF}_{6}, \mathrm{CF}_{3} \mathrm{I}$, hydrofluoroolefine, $\mathrm{He}+\mathrm{H}_{2} \mathrm{O}$ generated interest in swarm experiments because of their relevance to global warming, high voltage insulation, and biomedicine [15-18]. Particular transport effects [19-21] in specific gas mixtures (like negative differential conductivity) also motivate further experimental studies.

To obtain definite values of the transport coefficients it is important that measurements are conducted under 


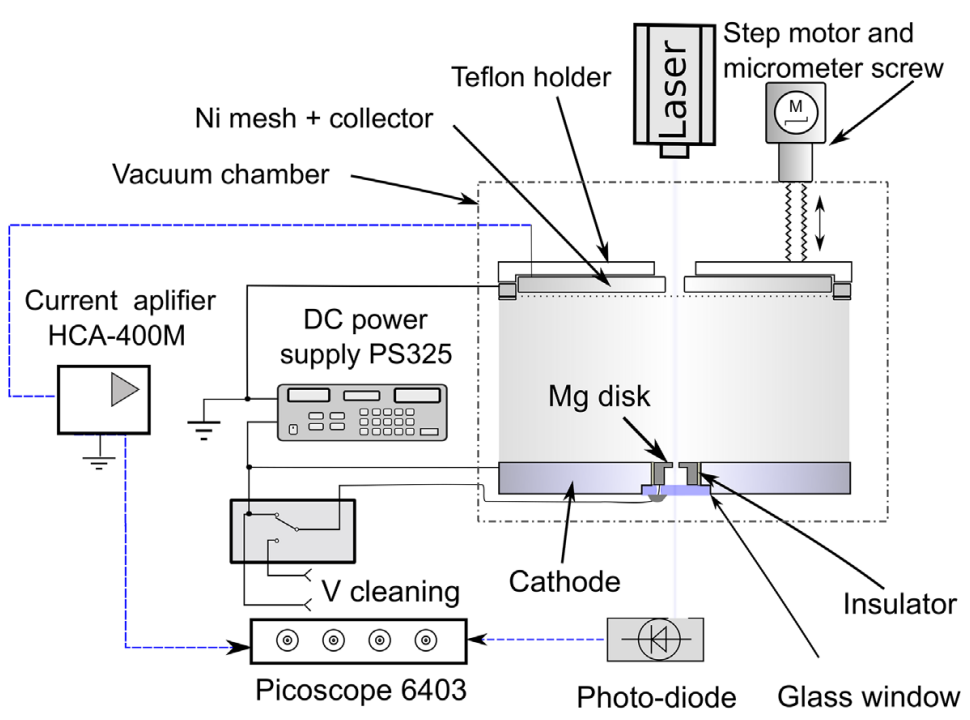

Figure 1. Simplified scheme of the experimental setup.

hydrodynamic conditions of the swarms, where the (reduced, i.e. density-rescaled) transport coefficients acquire unique values, which are functions only of the reduced electric field, $E / N$. In the hydrodynamic regime the space- and time-dependence of the electron density of a swarm generated at $t=0$ and $z=0$ is given as (see, e.g. [22]):

$$
n_{\mathrm{e}}(z, t)=\frac{n_{0}}{\left(4 \pi D_{\mathrm{L}} t\right)^{1 / 2}} \exp \left[\nu t-\frac{(z-W t)^{2}}{4 D_{\mathrm{L}} t}\right],
$$

where $n_{0}$ is the initial electron density, $D_{\mathrm{L}}$ is the (bulk) longitudinal diffusion coefficient, $\nu$ is the effective ionization frequency (equal to the ionization frequency minus the attachment frequency), and $W$ is the bulk drift velocity, defined as

$$
W=\frac{\mathrm{d}}{\mathrm{d} t}\left[\frac{\sum_{j=1}^{N_{\mathrm{e}}(t)} z_{j}(t)}{N_{\mathrm{e}}(t)}\right],
$$

where $N_{\mathrm{e}}(t)$ is the number of electrons in the swarm at time $t$. We note that it is important to distinguish the bulk drift velocity from the flux drift velocity, which is defined as

$$
w=\frac{\sum_{j=1}^{N_{\mathrm{e}}(t)} v_{j, z}(t)}{N_{\mathrm{e}}(t)},
$$

because these two quantities acquire different values in the presence of non-conservative processes, like ionization and attachment (see, e.g. [23]).

It is clear from (1) that the evolution of the electron density of the swarm is governed by the bulk drift velocity, and therefore it is this velocity that is accessible in swarm experiments based on the measurement of quantities related to $n_{\mathrm{e}}(z, t)$. The data acquisition procedures adopted in some experiments, however, yield velocities different from either of the (well-defined and well-understood) bulk and flux drift velocities (e.g. the so-called 'mean-arrival time drift velocity' [10]) - the complexity of the interpretation of the measured values has been discussed in detail, e.g. in [24] by Tagashira. (We note that in some publications no explicit reference is even given to the type of drift velocity values communicated.) In light of the above arguments, an ideal measurement and data acquisition approach should yield the bulk drift velocity, without any constraints on the effects of ionization/attachment and diffusion.

In [25] we have reported the development of a 'scanning' drift tube apparatus, which is capable of mapping the complete spatio-temporal evolution of electron swarms, developing between two plane electrodes (i.e. recording of 'swarm maps'). This system operates with electron swarms initiated by photoelectron pulses and the spatio-temporal distribution of the electron flux is recorded while the electrode gap length (at a fixed electric field strength and gas density) is varied (see section 2). The mapping approach used in this system allows direct identification of the nonhydrodynamic and hydrodynamic regions, which is important because measurements taken in the latter are clearly required to obtain well-defined transport coefficients. As it will be shown later, the measurements allow a transparent determination of 'time-of-flight' transport parameters: the (bulk) drift velocity $W$, the (reduced) longitudinal diffusion coefficient $N D_{\mathrm{L}}$, and the (reduced) effective ionization frequency $\nu / N$, from a common data set measured at fixed conditions. From these coefficients we also derive the (reduced) effective ionization coefficient $\alpha / N$, that characterizes the spatial growth of electron swarms under 'steadystate Townsend' conditions.

In section 2 of the paper the experimental apparatus and the data acquisition methods are described. Section 3 presents the experimentally determined transport coefficients, in comparison with those obtained in earlier works, for a number of gases: argon, synthetic air, methane and deuterium. Section 4 gives a brief summary of the work and an appendix provides the numerical values of the measured data. 


\section{Experimental apparatus and data evaluation}

The simplified scheme of our experimental setup is shown in figure 1 and its concise description is given below. For a more complete description of the system the reader is referred to [25].

The drift cell is situated inside a stainless steel vacuum chamber (shown only in a simplified manner in figure 1) that is pumped down (by a turbomolecular pump backed by a rotary pump) to a base pressure of $10^{-5} \mathrm{~Pa}$ for several days preceding the measurements. During the experiments-that have been conducted at a temperature of $T=293 \pm 2 \mathrm{~K}$ - the gas pressure inside the chamber is measured by a Pfeiffer CMR 362 capacitive gauge. The gases, which flow through the chamber at a small flow rate of the order of $1-5 \mathrm{sccm}$ (set by a mass flow controller), have at least 5.0 purity. In the case of deuterium the isotopic enrichment is $>99.8 \%$.

Our drift tube operates in pulsed mode, bunches of photoelectrons are emitted (induced by $1.7 \mu \mathrm{J}$-energy pulses of a frequency-quadrupled diode-pumped YAG laser operated at $\sim 3 \mathrm{kHz}$ repetition rate) from a $\mathrm{Mg}$ disk embedded into the negatively biased stainless steel cathode, situated at the bottom of the drift cell. The cathode voltage is provided by a PS-325 (Stanford Research Systems) power supply. The laser light reaches the $\mathrm{Mg}$ disk via a quartz window of the top flange of the vacuum chamber and via a $5 \mathrm{~mm}$ hole drilled into the top electrode. The $\mathrm{Mg}$ disk has a $0.6 \mathrm{~mm}$-diameter hole to allow part of the laser light to be detected by a fast photodiode placed underneath the cell, outside the vacuum chamber. The signal of this photodiode triggers the data collection.

A $105 \mathrm{~mm}$-diameter stainless steel, virtually grounded collector electrode is situated at the top of the cell. An electrically grounded nickel grid with $T=88 \%$ transmission and 45 lines/inch density is mounted at a fixed distance of $1 \mathrm{~mm}$ in front of the collector electrode. The collector and the grid are moved together by a stepping motor-driven micrometer screw mounted on a vacuum feedthrough of the vacuum chamber (see figure 1). The distance between the emitter (cathode) and the grid can be set within the range of $L=13.6-63.6 \mathrm{~mm}$ (we use $0.2-1 \mathrm{~mm}$ step size to map the development of the swarm). The electrodes are electrically insulated from the grounded chamber wall with Teflon sheaths.

In pulsed drift tubes equipped with only two electrodes ('pulsed Townsend' setup) the displacement current generated by all the charge carriers moving in the inter-electrode gap is sensed [7], which is proportional to the spatial integral of the electron density $n_{\mathrm{e}}(z, t)$ (provided that the particle transport is hydrodynamic). In our system, the signal at the collector, $I(z, t)$, is also generated by the displacement current, which is however, induced only by the electrons that enter the field free grid-collector gap. The contribution of any electron upon entering this region to the current signal generated at the collector is proportional to its velocity. At low pressures these electrons fly through this narrow $(1 \mathrm{~mm})$ gap quickly, while at high pressures their velocity becomes quickly randomized due to collisions. In either case the duration of the current pulse generated by a single incoming electron is very short. The superposition of a high number of such pulses generated by a high number of electrons entering the grid-collector gap is fed to a high speed current amplifier (type Femto HCA-400M) and is measured, synchronized with the photo-diode signal, by a digital oscilloscope (type Picoscope 6403B) with 0.8 ns time resolution.

The measured signal can be assumed to be proportional to the flux of the electrons entering the grid-collector gap. It follows that, as in the hydrodynamic regime the flux and the density are proportional, in this domain our system senses the $n_{\mathrm{e}}(z, t)$ density of the swarm, given by (1). Accordingly, the data shown in the form of 'swarm maps' in the next section can be interpreted as the electron density. (Note that the effect of the motion of ions is neglected due to the short time scales of interest here.)

The experiment is controlled by a computer using LabView software in sequential measurements. During the mapping sequence, the voltage is continuously adjusted to ensure that $E / N$ remains fixed for the different gap sizes. Data obtained for 3000-5000 pulses (depending on the signal intensity) are averaged at each position of the collector.

Scanning the electrode separation and recording the collector current allows us to obtain complete information about the spatial and temporal evolution of the electron swarms. The recording time of a swarm map, with an acceptable signal to noise ratio, takes typically $\approx 25-100 \mathrm{~min}$.

The values of $W, D_{\mathrm{L}}$, and $\nu$ can be obtained by fitting the function (1) - that includes these parameters - to the measured $I(z, t)$ data. In the fitting procedure, care has to be taken to limit the fitting region where hydrodynamic transport prevails, i.e. the swarm is fully equilibrated beyond a transient region near the source [26]. In our previous work [25] we have shown that the transient region-if present within the domain accessible in the measurements-is clearly observable in the swarm maps.

Having determined the 'time-of-flight' transport coefficients $W, D_{\mathrm{L}}$, and $\nu$, the effective Townsend ionization coefficient, $\alpha$, which is characteristic for steady-state Townsend experiments, is calculated as:

$$
\frac{1}{\alpha}=\frac{W}{2 \nu}+\sqrt{\left(\frac{W}{2 \nu}\right)^{2}-\frac{D_{\mathrm{L}}}{\nu}},
$$

based on the discussions in the papers of Tagashira [27] and of Blevin and Fletcher [28]. In the absence of diffusion (i.e. $D_{\mathrm{L}}=0$ ) equation (4) reduces to $\alpha=\nu / W$. This value is increased in the presence of diffusion, for the gases and conditions covered here this increase is between less than $1 \%$ and $20 \%$.

Besides the fitting of the whole theoretical and measured density distribution we also apply a 'slicing' method as another way to determine the bulk drift velocity. Cutting the $I(z, t)$ maps of the swarm, or the $n_{\mathrm{e}}(z, t)$ density of the swarm, given by (1), at fixed values of time results in symmetrical, Gaussian functions, and the peaks of these functions can 

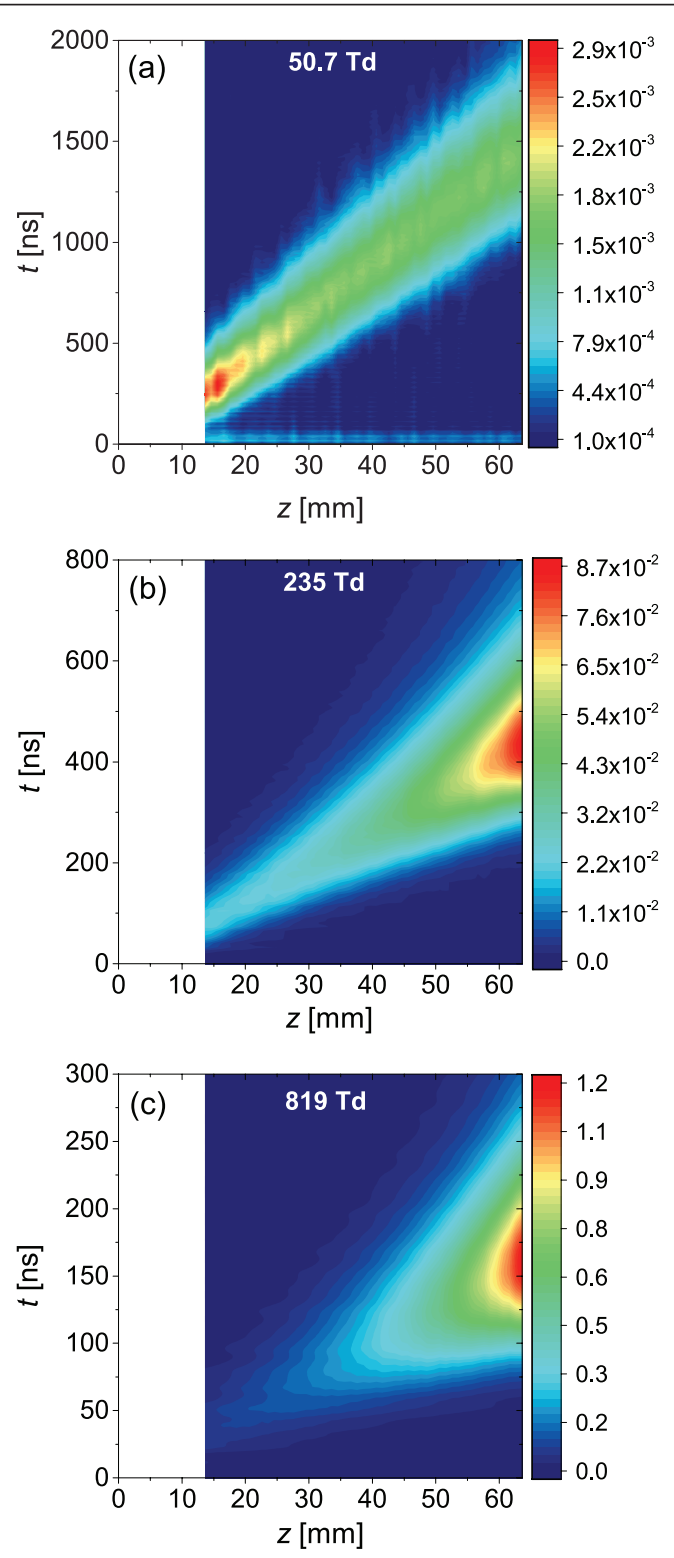

Figure 2. Swarm maps recorded in argon gas at reduced electric fields of (a) $50.7 \mathrm{Td}$, (b) $235 \mathrm{Td}$ and (c) $819 \mathrm{Td}$. Note the different time domains covered by the vertical axes. The color scales represent electron density and are given in arbitrary units, which are, however, the same for all panels.

be associated with the center-of-mass of the particle cloud. A straight line fit to the measured peak position as a function of time gives the value of $W$ directly (for more details see [25]).

The spatio-temporal distribution of the density of the swarm as given by (1) does not take into account transverse diffusion, which, however, is present in the experiment and can have an effect on the results under conditions when particles get lost at the cell wall (i.e. not all electrons arrive at the collector). To ensure that this effect-which may become important at long gaps - is not significant, we have executed the fitting of the measured signals to the theoretical form (1) for different subdomains of $z$ and compared the resulting transport coefficients. The results were accepted and are presented here only
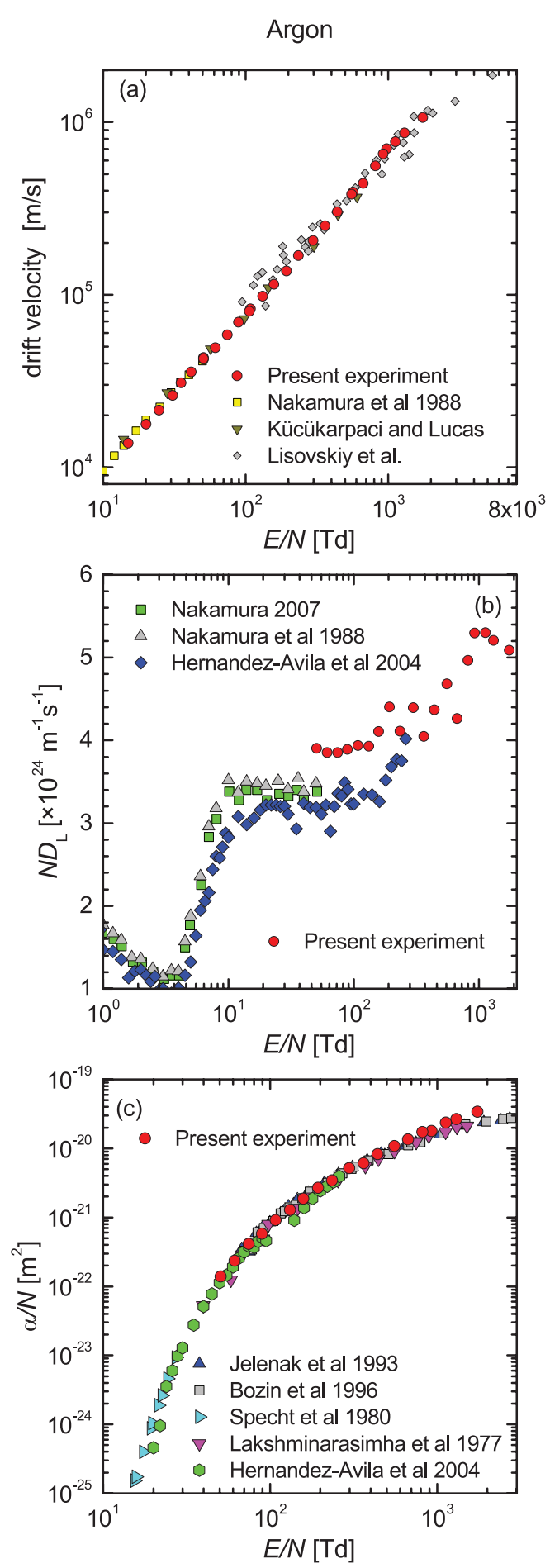

Figure 3. Electron transport coefficients in argon: (a) drift velocity, (b) reduced longitudinal diffusion coefficient and (c) reduced ionization coefficient. Previous data: Nakamura and Kurachi [29], Kücükarpaci and Lucas [30], Lisovskiy et al [31], Nakamura [32], HernándezÁvila et al [33], Jelenak et al [34], Bozin et al [35], Specht et al [36], Lakshminarasimha and Lucas [37]. Panel (a) [25] reprinted with permission AIP Publishing LLC. Copyright 2016 AIP Publishing LLC.

for those cases when the deviations between the data obtained from the fits over different $z$ domains were within $\pm 3 \%$ for $W$, $\pm 5 \%$ for $D_{\mathrm{L}}$ and $\nu$ ( $\pm 10 \%$ for synthetic air, due to the worse signal to noise ratio, see later). 


\section{Results}

In the following we present the experimental results for the different gases: argon, synthetic air, methane and deuterium. For each gas, representative examples are first given for the spatio-temporal swarm maps, and subsequently, we present the measured transport coefficients, in comparison with previous experimental data. We recall again that in previous works the definitions of the measured velocities differ and in some cases it is not even clear what kind of velocity was determined in a given experiment. Therefore we label the vertical axes of the corresponding figures as 'drift velocity' and discuss in the text the definitions used by the different authors, whenever this is possible.

For the determination of the bulk drift velocity $W$ we use both the slicing method and the fitting of the measured data to the theoretical $n_{\mathrm{e}}(z, t)$ profiles, described above. We find that at low $E / N$ the first method gives more accurate data, while at high $E / N$, where only a part of the Gaussian distributions is recorded, the fitting method has a better performance. (At low and medium $E / N$ values the two methods were found to yield drift velocities differing not more than $1 \%$.) For the determination of the longitudinal diffusion coefficient and the effective ionization frequency only the fitting of the complete distributions is used. Besides the graphical presentation of $W, N D_{\mathrm{L}}$ and $\alpha / N$, numerical values of these coefficients are also given in an appendix. The appendix contains as well values of $\nu / N$, which are not presented in the figures.

The accuracy of the transport coefficients is mainly limited by the noise present in the swarm maps (as will also be visible in the examples of $I(z, t)$ presented). At good signal to noise ratio the stability of the system ensured accurate reproducibility of the data, e.g. drift velocity values measured at the same conditions but on different days did not differ more than $2 \%$. The estimated errors of the results will be given at their presentation.

\subsection{Argon}

Exemplary spatio-temporal maps of electron swarms in argon gas are presented in figure 2, for different values of the reduced electric field, covering the range $E / N \sim 50 \mathrm{Td} \ldots 800 \mathrm{Td}$. At the lowest electric field traces of the equilibration of the swarm (as quasi-periodic structures) are observable at small gaps. At this low field $(50.7 \mathrm{Td})$ ionization is negligible and the spatial spreading of the electron cloud is rather low, indicating a small value of $D_{\mathrm{L}}$. A relatively high noise, characteristic of weak signals, measured, e.g. in the absence of appreciable ionization, is also seen here. At the higher electric fields the electron density grows significantly with the distance from the electron source, indicating the onset of significant ionization. At the higher fields $(E / N=235 \mathrm{Td}$ and $819 \mathrm{Td})$ the maps confirm that the swarms are already equilibrated within the non-accessible range of gap lengths $(z \leqslant 13.6 \mathrm{~mm})$. At the highest $E / N$ the amplitude of $I(z, t)$ reaches about 3 orders of magnitude higher values as compared to those recorded at the lowest $E / N$.
The electron transport coefficients in argon gas are presented in figure 3 . Our data for the measured bulk drift velocity of electrons, $W$-which cover a broader range of $E / N$ compared to any of the previous studies - agree well with those given by Nakamura and Kurachi [29] and Kücükarpaci and Lucas [30] at lower $E / N$ values and with the data of Lisovskiy et al [31] at higher $E / N$ (see figure 3(a)). We note that the latter data were derived from the radiofrequency breakdown characteristic of argon and not in a drift tube experiment, and have comparatively large scattering, unlike our data, which have an estimated error smaller than $3 \%$ for $E / N \leqslant 1000 \mathrm{Td}$, and $\sim 5 \%$ at $E / N>1000 \mathrm{Td}$.

The longitudinal diffusion coefficient $D_{\mathrm{L}}$ is presented in figure 3(b) together with values measured by Nakamura [32] in a double shutter drift tube below $50 \mathrm{Td}$, using a UV photoelectric source, results obtained by Nakamura and Kurachi [29] in a double shutter drift tube employing a filament electron source, as well as data provided by Hernández-Ávila et al [33] from measurements on a pulsed Townsend apparatus operated with $\mathrm{UV}\left(\mathrm{N}_{2}, 337 \mathrm{~nm}\right)$ laser pulses, covering a broader range of the reduced electric field, up to $E / N=400 \mathrm{Td}$. Our data extend the previously available values up to $E / N \approx 1000 \mathrm{Td}$, however, the scattering of the data is higher compared to the case of the drift velocity, as the fitting of $D_{\mathrm{L}}$ is more susceptible to the noise of the measured data. This results in an accuracy of $\pm 10 \%$ for our data for $D_{\mathrm{L}}$. Between $E / N=50 \mathrm{Td}$ and 200 Td our measurements indicate a $\approx 20 \%$ higher diffusion coefficient compared to the data of [33] (where a typical error of $D_{\mathrm{L}}$ was claimed to be $\left.10-15 \%\right)$.

The present values of the reduced ionization coefficient are shown in figure 3(c) in comparison with the data of Jelenak et al [34] and Bozin et al [35] who obtained the ionization and excitation coefficients for a wide range of the reduced electric field, $50 \mathrm{Td}-9 \mathrm{kTd}$, via measurements of the spatial dependence of the optical emission intensity from a stationary Townsend discharge (operated in the $\mu \mathrm{A}$ regime); Specht et al [36] who deduced the ionization coefficient from the ratio of the integrated ion and electron currents in a pulsed drifttube apparatus, and Lakshminarasimha and Lucas [37] who obtained $\alpha / N$ under stationary conditions, between 8 and 1271 $\mathrm{Td}$, based on the measurements of the current as a function of electrode gap in a drift tube. Our measured values (of which the accuracy we estimate to be below $6 \%$ ) cover a wider range of $E / N$, compared to the previous studies.

\subsection{Synthetic air}

Among all the gases, the lowest signal intensities and, consequently, the worst signal to noise ratios were found for synthetic air, most likely due to the modification of the photoelectron yield of the $\mathrm{Mg}$ disk by the gas itself. To achieve acceptable signal intensities the surface of the Mg disk had to be cleaned regularly by a sputtering discharge in Ar gas, which was possible using a separate electrical connection to the disk that could be connected as cathode without opening the vacuum chamber (for more details see [25]). This procedure resulted only in temporary enhancement of the signal, making it possible to measure up to five swarm maps with acceptable signal to noise ratio. 

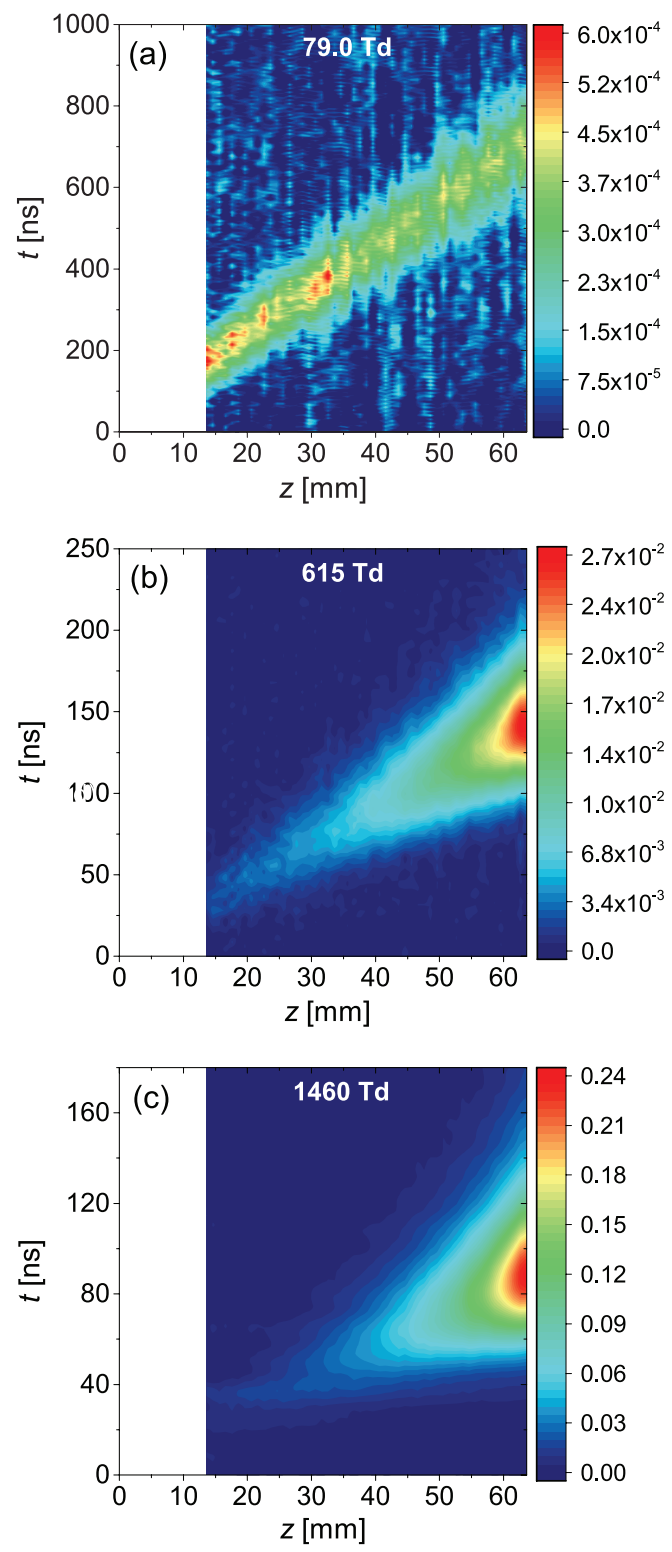

Figure 4. Swarm maps recorded in synthetic air, at reduced electric fields of (a) $79 \mathrm{Td}$, (b) $615 \mathrm{Td}$ and (c) $1460 \mathrm{Td}$. Note the different time domains covered by the vertical axes. The color scales represent electron density and are given in arbitrary units, which are, however, the same for all panels.

The poor signal to noise ratio is well visible in the swarm map recorded at the low value of $E / N=79 \mathrm{Td}$, see figure 4(a). With increasing $E / N$ the signal to noise ratio improves, but the quality of the data is still inferior compared to the other gases, which finally makes the determination of the transport parameters, especially the diffusion and ionization coefficients, less accurate and the results are limited to the high $E / N$ range.

The obtained values of the electron drift velocity in synthetic air are presented in figure 5(a). This figure includes data originating from several previous studies as well, that cover different domains of $E / N$. (Note that most of these previous measurements have been carried out using dry, but not
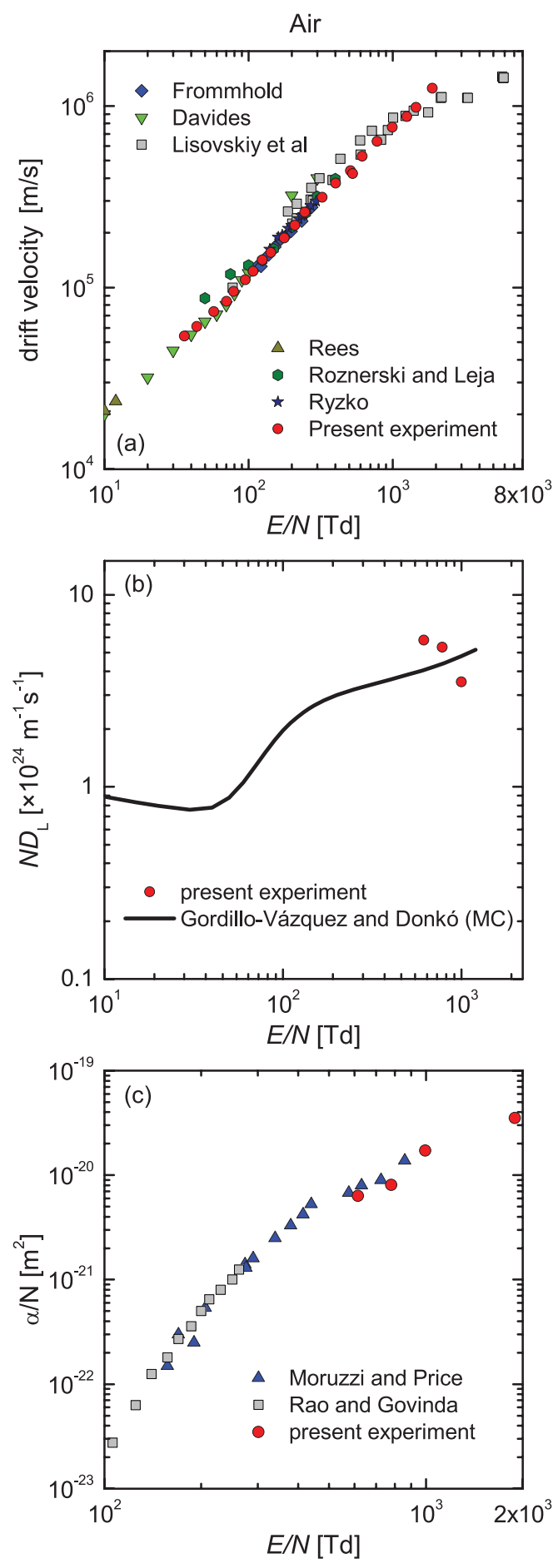

Figure 5. Electron transport coefficients in synthetic air: (a) drift velocity, (b) reduced longitudinal diffusion coefficient and (c) reduced effective ionization coefficient. Previous experimental and simulation data: Rees [38], Davies [39], Frommhold [40], Ryzko [41], Roznerski and Leja [42] and Lisovskiy and Yegorenkov [43], Gordillo-Vázquez and Donkó [46].

synthetic air. Minor constituents of dry air are, however, not expected to result in a significant change of the drift velocity.) The present data cover a wider domain of the reduced electric field than any of the previous works. All data sets agree 
reasonably well. At low $E / N$ the agreement with the data of Rees [38] and Davies [39] is quite good, as well as at medium $E / N$ values (between $100 \mathrm{Td}$ and $300 \mathrm{Td}$ ) with the data of Frommhold [40] and Ryzko [41]. Roznerski and Leja [42] used a double shutter time-of-flight setup for measuring the drift velocity for $E / N$ between 0.6 to $250 \mathrm{Td}$. The data of Lisovskiy and Yegorenkov [43] show comparatively more scattering, but we note, that as in the case of argon (see above), that their data were derived from the radiofrequency breakdown characteristic of the gas.

Experimental data for the longitudinal diffusion coefficient in air are difficult to find in the literature. Most studies have focused on the determination of the transverse diffusion coefficient only, see [44, 45] and references therein. Therefore, in figure 5(b) we include a simulation result for $D_{\mathrm{L}}$ obtained in [46], for comparison with our present data. Due to the weak signals in the experiment only a few data points (having an error up to $\pm 15 \%$ ) are given in figure 5 (b) for $D_{\mathrm{L}}$. For other (i.e. lower $E / N$ ) conditions even higher uncertainties were found and therefore the data are not presented.

The poor signal to noise in synthetic air also hindered the determination of the effective ionization coefficient in our measurements, data are only given for $E / N>600 \mathrm{Td}$. Nevertheless, the data agree reasonably with the values of $\alpha / N$ obtained in previous experiments of Raja Rao and Govinda Raju [47] as well as that of Moruzzi and Price [48], both based on measurements of the growth of a photoeffect-initiated current with gap length of their drift tubes. The uncertainty of our data for $\alpha / N$ is less than $10 \%$.

\subsection{Methane}

The widest range of $E / N$ with good signal to noise ratio was reached in methane, our measurements in this gas cover values from $1.5 \mathrm{Td}$ to $\sim 1500 \mathrm{Td}$. Illustrative examples of the measured swarm maps are shown in figure 6. At low E/N (figure 6(a)) the map indicates a negligible ionization and slow diffusion, the signal is concentrated within a narrow domain in space and time, i.e. the cloud practically does not spread while it travels with a well-defined velocity. For the case of the medium $E / N$ of $118 \mathrm{Td}$ (figure 6(b)) we find a decreasing peak intensity of the signal with increasing time and position, which is caused by the spreading of the electron cloud, due to the increased rate of diffusion at low ionization rate. At the highest reduced electric field of $1520 \mathrm{Td}$ we see the onset of ionization, there is a strong increase of the signal intensity as a function of position (see figure 6(c)).

The transport coefficients derived from the $I(z, t)$ swarm maps are displayed in figure 7 as a function of $E / N$, in comparison with data provided in earlier works. For comparison we include data from the following works. Al-Amin et al [49] used the TOF technique with a fixed drift gap length to obtain the drift velocity and the longitudinal diffusion coefficient within the range $0.28-848 \mathrm{Td}$; Davies et al [50] determined the transport coefficients of electron swarms initiated by UV light illumination, in a drift tube with variable gap length; Hunter et al [51] provided data for the drift velocity and the effective ionization coefficient using the pulsed Townsend technique; Schmidt and Roncossek [6] used two-photon ionization by intense UV laser light within the gas phase to create electron swarms and determined the drift velocity, as well as $D_{\mathrm{L}}$ and $D_{\mathrm{T}}$, at low reduced electric fields, limited to $E / N \leqslant 15 \mathrm{Td}$; Berghöfer et al [5] used as well UV laser ionization in the gas phase, however, in this experiment two laser beams were used to ionize the gas at two different positions, and the difference between the arrival times of the two particle clouds were measured; Yoshida et al [11] employed a double shutter drift tube and measured the arrival time 'spectrum' of electrons. The results obtained in all these experiments show a high degree of consistency, and a good agreement is also found with our present data, especially for the drift velocity values shown in figure 7(a). With regards to the longitudinal diffusion coefficient (see figure 7(b)) our data are most consistent with those of Al-Amin et al [49]: we find a 15\% higher minimum value for $D_{\mathrm{L}}$ at around $50 \mathrm{Td}$ compared to Yoshida et al [11], from whose values we also find deviations at $E / N$ values exceeding $200 \mathrm{Td}$-in this higher $E / N$ range our values are closest to those given by Al-Amin et al [49]. With regards to the effective ionization coefficient our measurements indicate slightly higher values as compared to other works around $E / N \sim 100 \mathrm{Td}$. At high $E / N$ values our data agree the best with Davies et al [50] and are 10-15\% lower than those given by Yoshida et al [11]. The uncertainties of the present values for $N D_{\mathrm{L}}$ and $\alpha / N$, are below $8 \%$ and $6 \%$, respectively.

\subsection{Deuterium}

Examples of recorded swarm maps for deuterium are shown in figure 8 , while the transport coefficients are given in figure 9 . We have also experienced for this gas a relatively poor signal to noise ratio at low $E / N$, which, however, improved significantly towards higher reduced electric fields.

Our drift velocity data are compared to the results of previous studies in figure 9(a). Petrović and Crompton [52] covered the 3-30 Td range of the reduced electric field and determined the drift velocity from the transit time of the swarm in a double shutter type drift tube. Roznerski et al [53] measured the drift velocity in a double shutter drift tube in the range of 3-125 Td. Values for low reduced electric fields $(E / N<15 \mathrm{Td})$ were also given by McIntosh [54]. While at low $E / N$ all data sets agree well, the present experiments indicate a noticeably higher drift velocity above $\sim 30 \mathrm{Td}$, compared to the data of Roznerski et al [53]. For $E / N$ values below $400 \mathrm{Td}$ the estimated error of our data for the drift velocity is below $3 \%$, and for high $E / N$ this grows up to $6 \%$.

The solid line labelled as 'Roznerski et al (calc)' in figure 9(a) was given in [53] as a result of a two-term solution of the Boltzmann equation with the cross sections by Buckman and Phelps [55]. While in [53] it was concluded that this cross section set may need to be adjusted, our present results are in a very good agreement with this calculated curve. 

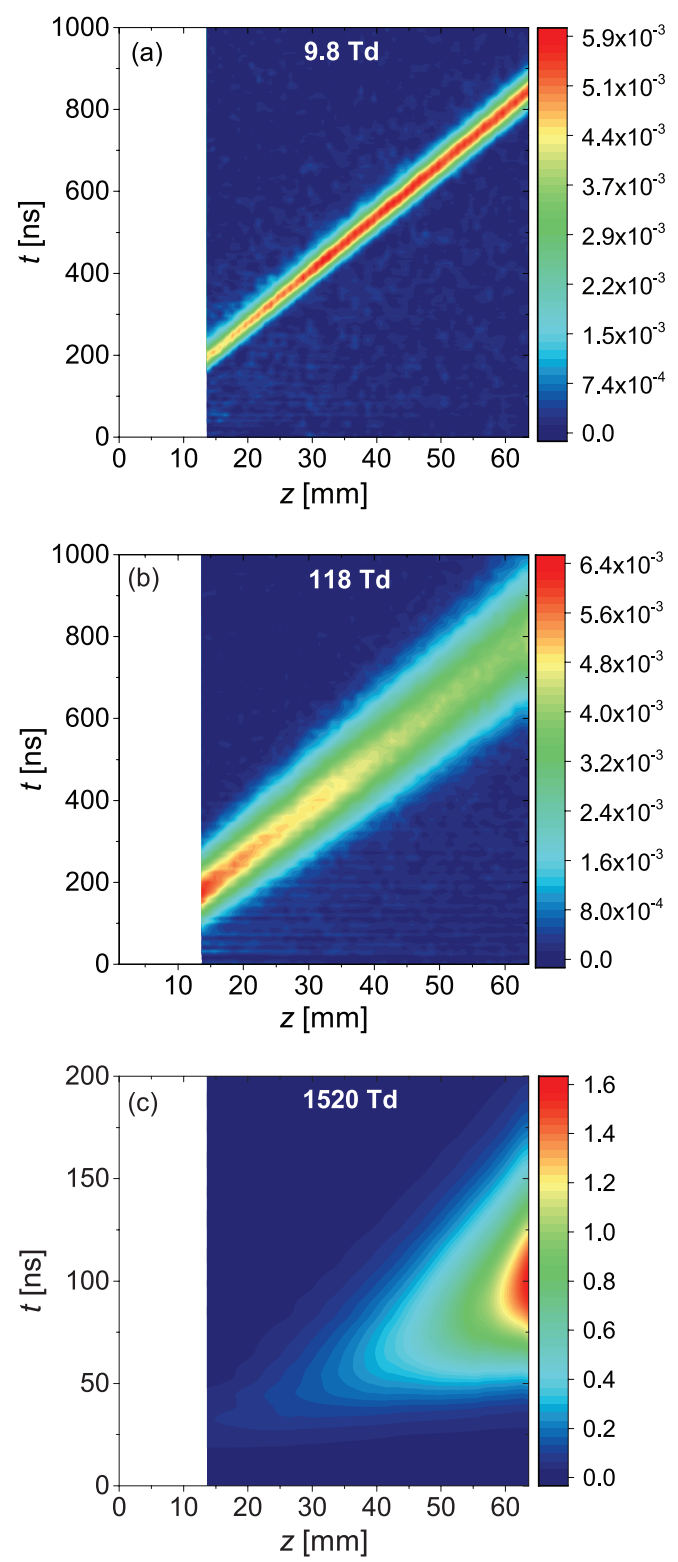

Figure 6. Swarm maps recorded in methane, at reduced electric fields of (a) $9.8 \mathrm{Td}$, (b) $118 \mathrm{Td}$ and (c) $1520 \mathrm{Td}$. Note the different time domains covered by the vertical axes. The color scales represent electron density and are given in arbitrary units, which are, however, the same for all panels.

We were able to find values for the longitudinal diffusion coefficient of electrons in $D_{2}$ only in the paper of McIntosh [54] (for low reduced electric fields, $E / N<6 \mathrm{Td}$ ). This set of data, together with the values measured in the present experiment are displayed in figure 9(b). The reduced ionization coefficient as a function of $E / N$, in comparison with values obtained by other authors is presented in figure 9(c). The data of Rose [56], Cowling and Fletcher [57], as well as Davies et al [58] were obtained via measurements of current growth in drift tubes with variable gap lengths. All data sets for $\alpha / N$, including ours, agree reasonably well over the whole domain of $E / N$. The error of our data for $D_{\mathrm{L}} N$ and $\alpha / N$ is estimated to be less than $10 \%$.
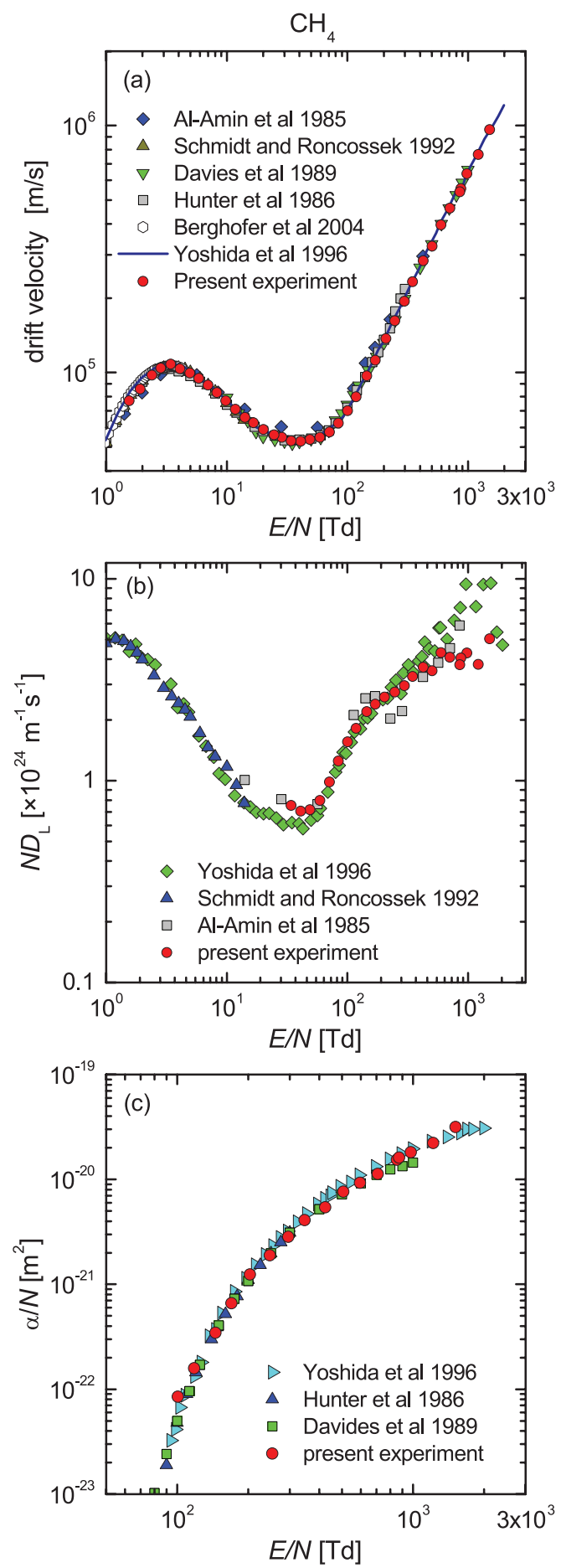

Figure 7. Electron transport coefficients in methane: (a) drift velocity, (b) reduced longitudinal diffusion coefficient and (c) reduced effective ionization coefficient. Previous data: Al-Amin et al [49], Davies et al [50], Hunter et al [51], Schmidt and Roncossek [6], Berghöfer et al [5], Yoshida et al [11].

\section{Summary}

A scanning drift tube apparatus, capable of mapping the complete spatial and temporal development of electron swarms has been employed to determine transport coefficients (bulk 

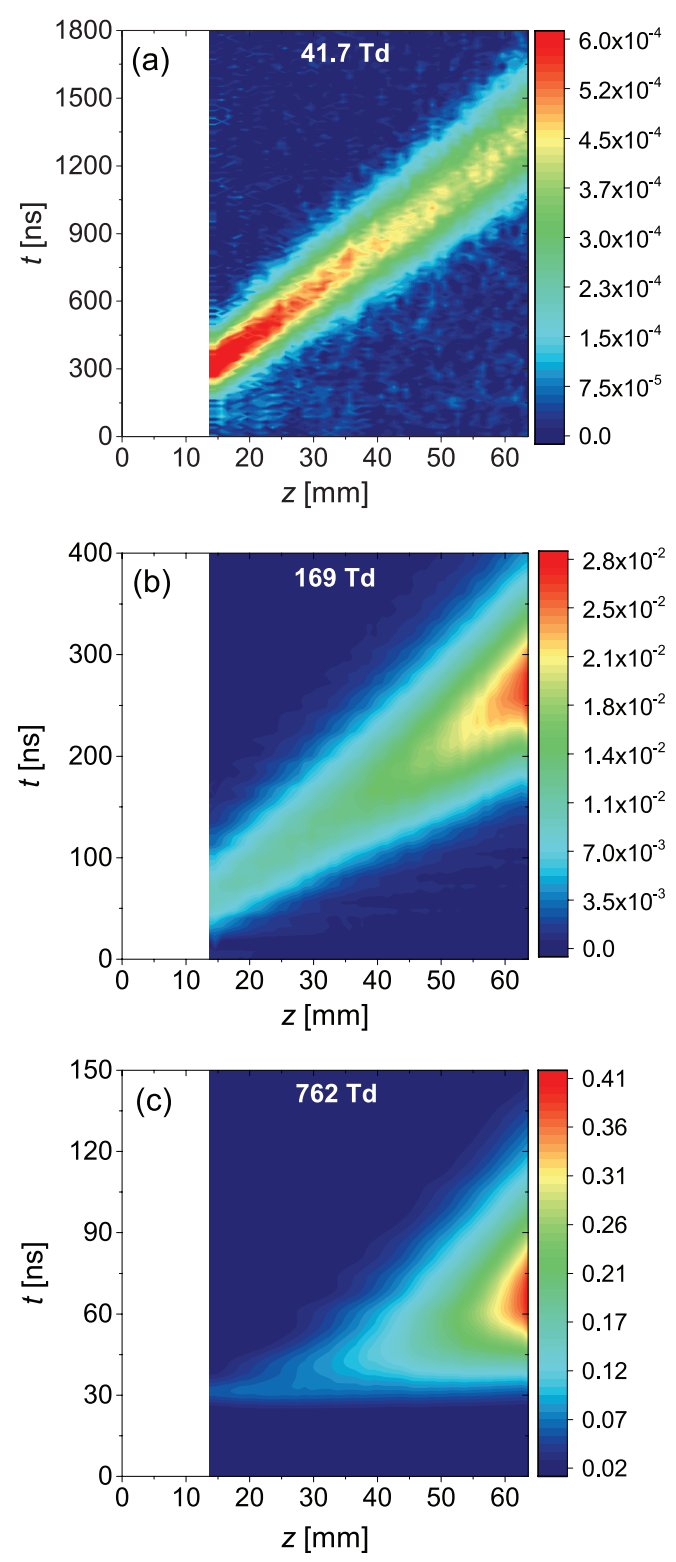

Figure 8. Swarm maps recorded in deuterium, at reduced electric fields of (a) $41.7 \mathrm{Td}$, (b) $169 \mathrm{Td}$ and (c) $762 \mathrm{Td}$. Note the different time domains covered by the vertical axes. The color scales represent electron density and are given in arbitrary units, which are, however, the same for all panels.

drift velocity, longitudinal diffusion coefficient, the effective ionization frequency and Townsend ionization coefficient) of electrons in different gases: argon, synthetic air, methane and deuterium. All three transport coefficients have been determined from the measured spatial and temporal distributions of the electron density, under hydrodynamic conditions, which are directly confirmed by the measured data, making calculations of the equilibration processes unnecessary. The transport coefficients determined in our experiments are given below in tabulated form in the appendix.

Our measurements have covered a wider range of $E / N$, or extended it (typically to higher $E / N$ values) compared to most of the previous investigations. For some conditions the poor signal to noise ratio has hindered the accurate determination
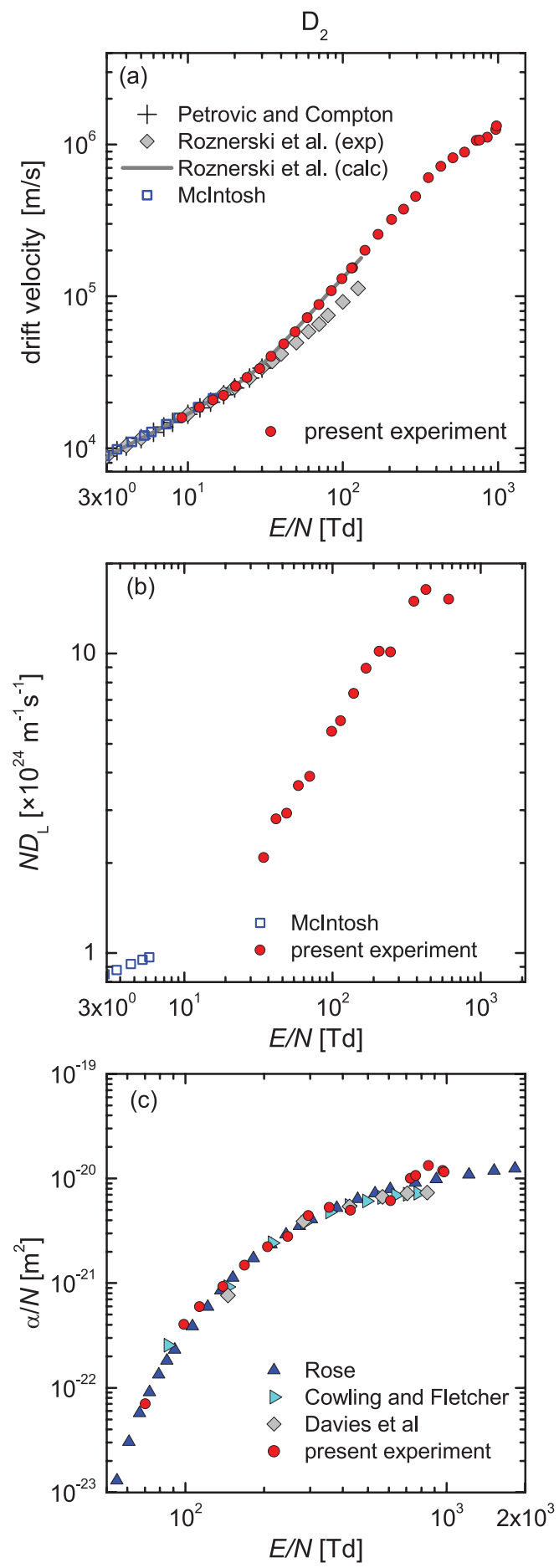

Figure 9. Electron transport coefficients in deuterium: (a) drift velocity, (b) reduced longitudinal diffusion coefficient and (c) reduced ionization coefficient. Previous data: Petrović and Crompton [52], Roznerski et al [53], McIntosh [54], Rose [56], Cowling and Fletcher [57], Davies et al [58].

of the transport coefficients (especially $D_{\mathrm{L}}$ ), application of a higher power UV laser source and/or a better electron emitter material could enhance the capabilities of our instrument. As an additional extension the collector electrode can be re-designed to consist of electrically separated rings, allowing the determination of the transverse diffusion coefficient, $D_{\mathrm{T}}$, as well. 
Table A1. Measured electron bulk drift velocity in different gases at $293 \mathrm{~K}$, as a function of the reduced electric field.

\begin{tabular}{|c|c|c|c|c|c|c|c|}
\hline \multicolumn{2}{|c|}{ Argon } & \multicolumn{2}{|c|}{ Synthetic air } & \multicolumn{2}{|c|}{ Methane } & \multicolumn{2}{|c|}{ Deuterium } \\
\hline $\begin{array}{l}E / N \\
(\mathrm{Td})\end{array}$ & $\begin{array}{l}W \\
\left(10^{4} \mathrm{~m} \mathrm{~s}^{-1}\right)\end{array}$ & $\begin{array}{l}E / N \\
(\mathrm{Td})\end{array}$ & $\begin{array}{l}W \\
\left(10^{4} \mathrm{~m} \mathrm{~s}^{-1}\right)\end{array}$ & $\begin{array}{l}E / N \\
(\mathrm{Td})\end{array}$ & $\begin{array}{l}W \\
\left(10^{4} \mathrm{~m} \mathrm{~s}^{-1}\right)\end{array}$ & $\begin{array}{l}E / N \\
(\mathrm{Td})\end{array}$ & $\begin{array}{l}W \\
\left(10^{4} \mathrm{~m} \mathrm{~s}^{-1}\right)\end{array}$ \\
\hline 15.01 & 1.38 & 36.01 & 5.40 & 1.552 & 7.70 & 9.155 & 1.59 \\
\hline 20.05 & 1.78 & 43.81 & 6.10 & 1.919 & 8.61 & 11.93 & 1.86 \\
\hline 24.74 & 2.15 & 57.54 & 7.35 & 2.397 & 9.77 & 14.52 & 2.08 \\
\hline 30.78 & 2.61 & 70.39 & 8.40 & 2.835 & 10.44 & 17.03 & 2.24 \\
\hline 35.31 & 3.09 & 78.99 & 9.50 & 3.426 & 10.84 & 20.32 & 2.57 \\
\hline 41.54 & 3.57 & 94.89 & 11.0 & 4.107 & 10.37 & 24.15 & 2.92 \\
\hline 50.75 & 4.23 & 107.6 & 12.3 & 4.944 & 9.98 & 29.08 & 3.34 \\
\hline 61.41 & 4.93 & 124.9 & 14.2 & 5.883 & 9.50 & 34.41 & 4.04 \\
\hline 74.73 & 5.87 & 143.3 & 15.6 & 6.978 & 8.93 & 41.65 & 4.87 \\
\hline 89.38 & 6.92 & 177.1 & 18.7 & 8.310 & 8.32 & 49.30 & 5.85 \\
\hline 106.5 & 8.03 & 209.8 & 22.0 & 9.700 & 7.71 & 59.10 & 7.26 \\
\hline 108.4 & 8.30 & 245.9 & 26.0 & 11.76 & 7.12 & 70.27 & 8.83 \\
\hline 131.9 & 9.79 & 325.3 & 31.4 & 14.19 & 6.59 & 84.17 & 10.9 \\
\hline 158.6 & 11.5 & 403.3 & 37.5 & 16.61 & 6.28 & 98.82 & 13.1 \\
\hline 193.8 & 13.7 & 510.5 & 43.9 & 20.12 & 5.90 & 113.8 & 15.4 \\
\hline 235.3 & 16.8 & 528.6 & 42.3 & 24.72 & 5.60 & 115.9 & 15.5 \\
\hline 298.7 & 20.7 & 614.6 & 52.8 & 28.62 & 5.49 & 139.2 & 20.1 \\
\hline 362.8 & 25.1 & 781.1 & 63.7 & 34.24 & 5.31 & 168.7 & 25.7 \\
\hline 440.7 & 30.3 & 996.5 & 76.1 & 40.98 & 5.29 & 206.5 & 32.1 \\
\hline 554.8 & 38.2 & 1260 & 87.4 & 48.86 & 5.37 & 246.4 & 37.5 \\
\hline 569.0 & 39.3 & 1459 & 98.0 & 59.18 & 5.48 & 295.8 & 45.7 \\
\hline 670.9 & 44.1 & 1889 & 125 & 71.05 & 5.76 & 356.0 & 60.5 \\
\hline 818.6 & 55.9 & & & 84.09 & 6.26 & 429.2 & 71.9 \\
\hline 926.5 & 65.6 & & & 100.8 & 7.02 & 513.0 & 82.0 \\
\hline 979.2 & 70.1 & & & 118.3 & 7.99 & 609.8 & 89.3 \\
\hline 1129 & 77.0 & & & 145.3 & 9.71 & 726.0 & 106 \\
\hline 1308 & 86.8 & & & 170.2 & 11.3 & 762.7 & 107 \\
\hline 1750 & 106 & & & 208.8 & 13.8 & 852.6 & 112 \\
\hline & & & & 246.9 & 16.2 & 966.9 & 126 \\
\hline & & & & 296.5 & 19.5 & 978.6 & 133 \\
\hline & & & & 346.83 & 23.4 & & \\
\hline & & & & 424.8 & 28.4 & & \\
\hline & & & & 505.8 & 32.6 & & \\
\hline & & & & 596.5 & 39.7 & & \\
\hline & & & & 708.6 & 46.4 & & \\
\hline & & & & 854.2 & 54.0 & & \\
\hline & & & & 872.4 & 55.7 & & \\
\hline & & & & 978.6 & 64.1 & & \\
\hline & & & & 1219 & 76.4 & & \\
\hline & & & & 1516 & 96.6 & & \\
\hline
\end{tabular}

\section{Acknowledgments}

This work was supported by the Hungarian Fund for Scientific Research (OTKA), via grant K105476. We gratefully acknowledge the contributions of T Szúcs and P Hartmann to the development of the experimental apparatus. We thank N Pinhão and D Loffhagen for useful discussions.

\section{Appendix}

The tables A1-A4 below give numerical values of the electron transport coefficients obtained in our measurements.

\section{References}

[1] Petrović Z Lj, Šuvakov M, Nikitović Ž, Dujko S, Šašić O, Jovanović J, Malović G and Stojanović V 2007 Plasma Sources Sci. Technol. 16 S1

[2] Elford M T 1972 In Case Studies in Atomic and Collision Physics vol 2, ed E W McDaniel and M R C McDowell (Amsterdam: North-Holland) ch 2

[3] Huxley L G H and Crompton R W 1974 The Diffusion and Drift of Electrons in Gases (New York: Wiley)

[4] Crompton R W 1994 Advances in Atomic, Molecular and Optical Physics vol 33, ed M Inokuti (New York: Academic)

[5] Berghöfer Th, Blümer J and Hörandel J R 2004 Nucl. Instrum. Methods Phys. A 525544 
Table A2. Measured reduced longitudinal diffusion coefficient of electrons in different gases at $293 \mathrm{~K}$, as a function of the reduced electric field.

\begin{tabular}{|c|c|c|c|c|c|c|c|}
\hline \multicolumn{2}{|r|}{ Argon } & \multicolumn{2}{|c|}{ Synthetic air } & \multicolumn{2}{|c|}{ Methane } & \multicolumn{2}{|c|}{ Deuterium } \\
\hline $\begin{array}{l}E / N \\
(\mathrm{Td})\end{array}$ & $\begin{array}{l}N D_{L} \\
\left(10^{24} \mathrm{~m}^{-1} \mathrm{~s}^{-1}\right)\end{array}$ & $\begin{array}{l}E / N \\
(\mathrm{Td})\end{array}$ & $\begin{array}{l}N D_{L} \\
\left(10^{24} \mathrm{~m}^{-1} \mathrm{~s}^{-1}\right)\end{array}$ & $\begin{array}{l}E / N \\
(\mathrm{Td})\end{array}$ & $\begin{array}{l}N D_{L} \\
\left(10^{24} \mathrm{~m}^{-1} \mathrm{~s}^{-1}\right)\end{array}$ & $\begin{array}{l}E / N \\
(\mathrm{Td})\end{array}$ & $\begin{array}{l}N D_{L} \\
\left(10^{24} \mathrm{~m}^{-1} \mathrm{~s}^{-1}\right)\end{array}$ \\
\hline 50.75 & 3.91 & 614.6 & 5.79 & 34.24 & 0.755 & 34.41 & 2.09 \\
\hline 61.41 & 3.86 & 781.1 & 5.33 & 40.98 & 0.709 & 41.65 & 2.81 \\
\hline 74.73 & 3.85 & 996.5 & 3.52 & 48.86 & 0.719 & 49.30 & 2.94 \\
\hline 89.38 & 3.89 & & & 59.19 & 0.800 & 59.10 & 3.63 \\
\hline 108.4 & 3.94 & & & 71.10 & 0.986 & 70.27 & 3.90 \\
\hline 131.9 & 3.93 & & & 84.09 & 1.25 & 98.82 & 5.51 \\
\hline 158.6 & 4.11 & & & 100.8 & 1.56 & 113.3 & 5.97 \\
\hline 193.8 & 4.40 & & & 118.3 & 1.82 & 139.2 & 7.38 \\
\hline 235.3 & 4.11 & & & 145.3 & 2.20 & 168.7 & 8.94 \\
\hline 298.7 & 4.40 & & & 170.2 & 2.40 & 206.5 & 10.2 \\
\hline 362.8 & 4.05 & & & 203.9 & 2.60 & 246.4 & 10.2 \\
\hline 440.7 & 4.37 & & & 246.9 & 2.76 & 355.9 & 15.0 \\
\hline 554.8 & 4.68 & & & 296.5 & 2.97 & 429.2 & 16.4 \\
\hline 670.8 & 4.27 & & & 346.8 & 3.29 & 609.8 & 15.3 \\
\hline 818.6 & 4.97 & & & 424.8 & 3.67 & & \\
\hline 926.5 & 5.30 & & & 505.8 & 3.51 & & \\
\hline \multirow[t]{7}{*}{1129} & 5.30 & & & 596.5 & 4.31 & & \\
\hline & & & & 708.6 & 4.10 & & \\
\hline & & & & 854.2 & 3.76 & & \\
\hline & & & & 872.4 & 4.06 & & \\
\hline & & & & 978.6 & 4.30 & & \\
\hline & & & & 1219 & 3.77 & & \\
\hline & & & & 1516 & 5.07 & & \\
\hline
\end{tabular}

Table A3. Measured reduced effective ionization frequency in different gases at $293 \mathrm{~K}$, as a function of the reduced electric field.

\begin{tabular}{|c|c|c|c|c|c|c|c|}
\hline \multicolumn{2}{|c|}{ Argon } & \multicolumn{2}{|c|}{ Synthetic air } & \multicolumn{2}{|c|}{ Methane } & \multicolumn{2}{|c|}{ Deuterium } \\
\hline $\begin{array}{l}E / N \\
(\mathrm{Td})\end{array}$ & $\begin{array}{l}\nu / N \\
\left(10^{-14} \mathrm{~m}^{3} \mathrm{~s}^{-1}\right)\end{array}$ & $\begin{array}{l}E / N \\
(\mathrm{Td})\end{array}$ & $\begin{array}{l}\nu / N \\
\left(10^{-14} \mathrm{~m}^{3} \mathrm{~s}^{-1}\right)\end{array}$ & $\begin{array}{l}E / N \\
(\mathrm{Td})\end{array}$ & $\begin{array}{l}\nu / N \\
\left(10^{-14} \mathrm{~m}^{3} \mathrm{~s}^{-1}\right)\end{array}$ & $\begin{array}{l}E / N \\
(\mathrm{Td})\end{array}$ & $\begin{array}{l}\nu / N \\
\left(10^{-14} \mathrm{~m}^{3} \mathrm{~s}^{-1}\right)\end{array}$ \\
\hline 24.74 & 0.00002 & 614.6 & 0.307 & 100.8 & 0.0006 & 70.27 & 0.0006 \\
\hline 30.78 & 0.00006 & 781.1 & 0.445 & 118.3 & 0.0013 & 98.82 & 0.0051 \\
\hline 35.31 & 0.00011 & 996.5 & 1.21 & 145.3 & 0.0033 & 113.3 & 0.0091 \\
\hline 41.54 & 0.00025 & 1889 & 3.65 & 170.2 & 0.0074 & 139.2 & 0.0174 \\
\hline 50.75 & 0.00059 & & & 203.9 & 0.017 & 168.7 & 0.035 \\
\hline 61.41 & 0.0014 & & & 246.9 & 0.030 & 206.5 & 0.067 \\
\hline 74.75 & 0.0024 & & & 296.5 & 0.053 & 246.4 & 0.097 \\
\hline 89.38 & 0.0039 & & & 346.8 & 0.090 & 295.8 & 0.185 \\
\hline 108.4 & 0.0073 & & & 424.8 & 0.142 & 355.9 & 0.282 \\
\hline 131.9 & 0.012 & & & 505.8 & 0.228 & 429.2 & 0.318 \\
\hline 158.6 & 0.020 & & & 596.5 & 0.332 & 609.8 & 0.468 \\
\hline 193.8 & 0.034 & & & 708.6 & 0.450 & 726.0 & 0.940 \\
\hline 235.3 & 0.053 & & & 854.2 & 0.698 & 762.6 & 0.995 \\
\hline 298.7 & 0.095 & & & 872.4 & 0.761 & 852.6 & 1.28 \\
\hline 362.8 & 0.139 & & & 978.6 & 0.973 & 966.9 & 1.36 \\
\hline 440.7 & 0.220 & & & 1219 & 1.38 & 978.6 & 1.35 \\
\hline 554.8 & 0.371 & & & 1516 & 2.56 & & \\
\hline 670.8 & 0.518 & & & & & & \\
\hline 818.6 & 0.817 & & & & & & \\
\hline 926.5 & 1.08 & & & & & & \\
\hline 1129 & 1.53 & & & & & & \\
\hline
\end{tabular}


Table A4. Measured reduced Townsend ionization coefficient in different gases at $293 \mathrm{~K}$, as a function of the reduced electric field.

\begin{tabular}{|c|c|c|c|c|c|c|c|}
\hline \multicolumn{2}{|c|}{ Argon } & \multicolumn{2}{|c|}{ Synthetic air } & \multicolumn{2}{|c|}{ Methane } & \multicolumn{2}{|c|}{ Deuterium } \\
\hline $\begin{array}{l}E / N \\
(\mathrm{Td})\end{array}$ & $\begin{array}{l}\alpha / N \\
\left(10^{-20} \mathrm{~m}^{2}\right)\end{array}$ & $\begin{array}{l}E / N \\
(\mathrm{Td})\end{array}$ & $\begin{array}{l}\alpha / N \\
\left(10^{-20} \mathrm{~m}^{2}\right)\end{array}$ & $\begin{array}{l}E / N \\
(\mathrm{Td})\end{array}$ & $\begin{array}{l}\alpha / N \\
\left(10^{-20} \mathrm{~m}^{2}\right)\end{array}$ & $\begin{array}{l}E / N \\
(\mathrm{Td})\end{array}$ & $\begin{array}{l}\alpha / N \\
\left(10^{-20} \mathrm{~m}^{2}\right)\end{array}$ \\
\hline 24.74 & 0.0007 & 614.6 & 0.64 & 100.8 & 0.009 & 70.27 & 0.007 \\
\hline 30.78 & 0.0023 & 781.1 & 0.81 & 118.3 & 0.016 & 98.82 & 0.041 \\
\hline 35.31 & 0.0036 & 996.5 & 1.72 & 145.3 & 0.035 & 113.3 & 0.060 \\
\hline 41.54 & 0.0070 & 1889 & 3.51 & 170.2 & 0.067 & 139.2 & 0.094 \\
\hline 50.75 & 0.014 & & & 203.9 & 0.125 & 168.7 & 0.150 \\
\hline 61.41 & 0.024 & & & 246.9 & 0.191 & 206.5 & 0.225 \\
\hline 74.75 & 0.038 & & & 296.5 & 0.286 & 246.4 & 0.281 \\
\hline 89.38 & 0.058 & & & 346.8 & 0.413 & 295.8 & 0.445 \\
\hline 108.4 & 0.091 & & & 424.8 & 0.544 & 355.9 & 0.531 \\
\hline 131.9 & 0.128 & & & 505.8 & 0.771 & 429.2 & 0.500 \\
\hline 158.6 & 0.188 & & & 596.5 & 0.937 & 609.8 & 0.618 \\
\hline 193.8 & 0.267 & & & 708.6 & 1.14 & 726.0 & 1.01 \\
\hline 235.3 & 0.345 & & & 854.2 & 1.55 & 762.6 & 1.07 \\
\hline 298.7 & 0.519 & & & 872.4 & 1.63 & 852.6 & 1.33 \\
\hline 362.8 & 0.614 & & & 978.6 & 1.82 & 966.9 & 1.20 \\
\hline 440.7 & 0.825 & & & 1219 & 2.24 & 978.6 & 1.16 \\
\hline 554.8 & 1.08 & & & 1516 & 3.18 & & \\
\hline 670.8 & 1.35 & & & & & & \\
\hline 818.6 & 1.73 & & & & & & \\
\hline 926.5 & 1.79 & & & & & & \\
\hline 1129 & 2.38 & & & & & & \\
\hline
\end{tabular}

[6] Schmidt B and Roncossek M 1992 Aust. J. Phys. 45351

[7] Raether H 1964 Electron Avalanches and Breakdown in Gases (London: Butterworths)

[8] de Urquijo J, Arriaga C A, Cisneros C and Alvarez I 1999 J. Phys. D: Appl. Phys. 3241

[9] Dahl D A, Teich T H and Franck C M 2012 J. Phys. D: Appl. Phys. 45485201

[10] Kondo K and Tagashira H 1990 J. Phys. D: Appl. Phys. 231175

[11] Yoshida K, Ohshima T Ohmori Y, Ohuchi H and Tagashira H 1995 J. Phys. D: Appl. Phys. 291209

[12] Dengming X and Yunkun D 2013 Plasma Sources Sci. Technol. 1525

[13] de Urquijo J, Juárez A M, Basurto E and Hernándes-Ávila J L 2007 J. Phys. D: Appl. Phys 402205

[14] Yoshida K, Ohshima T, Ohuchi H, Kishimoto Y and Tagashira H 1996 J. Phys. D: Appl. Phys 292447

[15] Christophorou L G and Olthoff J K 2000 J. Phys. Chem. Ref. Data 29267

[16] de Urquijo J, Mitrani A, Ruiz-Vargas G and Basurto E 2011 J. Phys. D: Appl. Phys 44342001

[17] Chachereau A, Rabie M and Franck C M 2016 Plasma Sources Sci. Technol. 25045005

[18] de Urquijo J, Basurto E, Juárez A M, Ness K F, Brunger M J and White R D 2014 J. Chem. Phys. 141014308

[19] Šašić O, de Urquijo J, Juárez A M, Dupljanin S, Jovanović J, Hernández-Ávila J L, Basurto E and Petrović Z Lj 2010 Plasma Sources Sci. Technol. 19034003

[20] de Urquijo J 2002 Plasma Sources Sci. Technol. 11 A86

[21] Dyatko N A, Kochetov I V and Napartovich A P 2014 Plasma Sources Sci. Technol. 23043001

[22] Robson R E 1991 Aust. J. Phys. 44685

[23] Dujko S, White R D and Petrović Z Lj 2008 J. Phys. D: Appl. Phys. 41245205

[24] Tagashira H 1992 Aust. J. Phys. 45365

[25] Korolov I, Vass M, Bastykova N Kh and Donkó Z 2016 Rev. Sci. Instrum. 87063102
[26] Malović G, Strinić A, Živanov A, Marić D and Petrović Z Lj 2003 Plasma Sources Sci. Technol. 12 S1

Donkó Z 2011 Plasma Sources Sci. Technol. 20024001

[27] Tagashira H, Sakai Y and Sakamoto S 1977 J. Phys. D: Appl. Phys. 101051

[28] Blevin H A and Fletcher J 1984 Aust. J. Phys. 37593

[29] Nakamura Y and Kurachi M 1988 J. Phys. D: Appl. Phys. 21718

[30] Kücükarpaci H N and Lucas J 1981 J. Phys. D: Appl. Phys. 142001

[31] Lisovskiy V A 1998 Tech. Phys. Lett. 24308 Lisovskiy V, Booth J-P, Landry K, Douai D, Cassagne V and Yegorenkov V 2006 J. Phys. D: Appl. Phys. 39660

[32] Nakamura Y 2007 Proc. of 28th ICPIG pp 224-6

[33] Hernandez-Avila J L, Basurto E and de Urquijo J 2004 J. Phys. D: Appl. Phys. 373088

[34] Jelenak Z M, Velikić Z B, Božin J V, Petrović Z Lj and Jelenković B M 1993 Phys. Rev. E 473566

[35] Božin J V, Jelenak Z M, Velikić Z V, Belča I D, Petrović Z Lj and Jelenković B M 1996 Phys. Rev. E 534007

[36] Specht L T, Lawton S A and DeTemple T A 1980 J. Appl. Phys. 51166

[37] Lakshminarasimha C S and Lucas J 1977 J. Phys. D: Appl. Phys. 10313

[38] Rees J A 1973 Aust. J. Phys. 26427

[39] Davies D K 1983 Theoretical Notes, Note 346 (Pittsburgh, PA: Westinghouse RD Center)

[40] Frommhold L 1963 Fortschr. Phys. 12597

[41] Ryzko H 1965 Proc. Phys. Soc. Lond. 85128

[42] Roznerski W and Leja K 1984 J. Phys. D: Appl. Phys. 17279

[43] Lisovskiy V A and Yegorenkov V D 1998 J. Phys. D: Appl. Phys. 313349

[44] Rees J A and Jory R L 1971 Aust. J. Phys. 17307

[45] Raja Rao C and Govinda Raju G R 1971 J. Phys. D: Appl. Phys. 4769

[46] Gordillo-Vázquez F J and Donkó Z 2009 Plasma Sources Sci. Technol. 18034021 
[47] Raja Rao C and Govinda Raju G R 1971 J. Phys. D: Appl. Phys. 4494

[48] Moruzzi J L and Price D A 1974 J. Phys. D: Appl. Phys 71434

[49] Al-Amin S A J, Kücükarpaci H N and Lucas J 1985 J. Phys. D: Appl. Phys. 181781

[50] Davies D K, Kline L E and Bies W E 1989 J. Appl. Phys. 653311

[51] Hunter S R, Carter J G and Christophorou L G 1986 J. Appl. Phys. 6024
[52] Petrović Z Lj and Crompton R W 1992 Aust. J. Phys. 42609

[53] Roznerski W, Mechlińsa-Drewko J, Leja K and Petrović Z Lj 1994 J. Phys. D: Appl. Phys. 272060

[54] McIntosh A I 1966 Aust. Phys. 19805

[55] Buckman S J and Phelps A V 1985 J. Chem. Phys. 824999

[56] Rose D J 1956 Phys. Rev. 104273

[57] Cowling I R and Fletcher J 1973 J. Phys. B: At. Mol. Phys. 6665

[58] Davies D E, Smith D and Myatt J 1961 Proc. V Int. Conf. Ion Phenom. in Gases (Munich) vol 1 p 678 